H I G H L I G H T S

TUMORIGENESIS

\section{RapTOR strikes again}

Rapamycin, the immunosuppressant inhibitor of the kinase TOR, is in clinical trials for the treatment of several different cancers, but the exact mechanism by which this drug prevents tumour growth is unclear. In the June issue of Nature Medicine, William Sellers and colleagues show that a rapamycin analogue inhibits prostate tumour growth by two independent pathways in mice - induction of mitochondrial-mediated apoptosis and inhibition of proliferation through the downregulation of the transcription factor Hif-1.

TOR kinase activity can be regulated by the oncogenic serine/threonine kinase AKT. When targeted to the mouse ventral prostate, transgenic Akt drives the development of prostate intra-epithelial neoplasia. Sellers and colleagues show that in these mice complete inhibition of Tor activity by the oral rapamycin derivative RAD001 induces a return to normal cell morphology within the intraluminal tissue of the prostate epithelium. The authors noted not only an increase in apoptotic cells within the regressing tumours, but also a concomitant inhibition of proliferation.

So how does Tor inhibition both induce apoptosis and inhibit proliferation? The authors addressed this by crossing the transgenic-Akt mice with mice specifically expressing human BCL2 in the ventral prostate. BCL2 prevented the apoptotic death of cells following treatment with the rapamycin

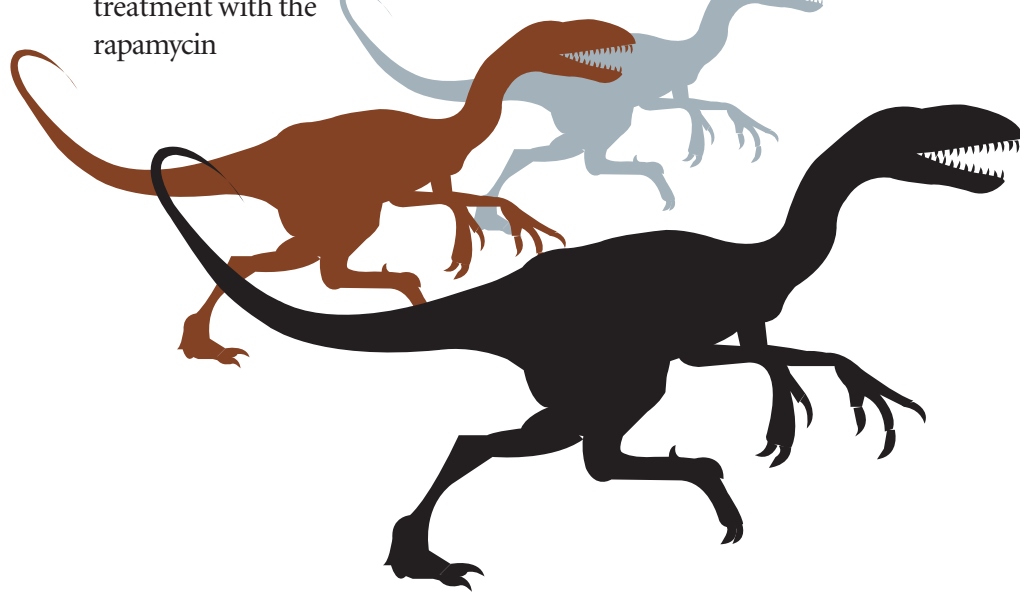

analogue, indicating that the mitochondrial apoptosis pathway is important for part of the rapamycin-mediated efficacy in this tumour model. However, BCL2 expression did not reverse the rapamycin-mediated inhibition of proliferation.

Which genes drive Akt-mediated proliferation? The authors used an intricate series of microarray- and computer-based technologies to show that the hypoxia-responsive protein Hif- $1 \alpha$ is upregulated in both the Aktand Akt/BCL2-expressing tumours and that its activity is suppressed in RAD001-treated mice. Although others have published links between HIF-1 $\alpha$, TOR and AKT previously, the mechanism by which TOR regulates HIF- $1 \alpha$ activity remains unclear.

Data from this mouse model indicates that human prostate cancer cells that overexpress BCL2 or that have TORindependent activation of HIF- $1 \alpha$ could be resistant to TOR inhibitors. More importantly, the current evaluation of RAD001 in patients with advanced prostate cancer might not prove informative without a corresponding analysis of tumour genotype.

Nicola McCarthy

(9) References and links ORIGINAL RESEARCH PAPER Majumder, P. K. et al. mTOR inhibition reverses AKT-dependent prostate intraepithelial neoplasia through regulation of apoptotic and Hif1 dependent pathways. Nature Med. 10, 594-601 (2004) FURTHER READING Denmeade, S. R. \& Isaacs, J. T. A history of prostate cancer treatment. Nature Rev. Cancer 2, 389-396 (2002)

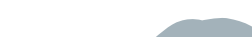$$
\text { (a) }
$$ 